\title{
Review Article \\ Employing Endogenous NSCs to Promote Recovery of Spinal Cord Injury
}

\author{
Sumei Liu ${ }^{1,2}$ and Zhiguo Chen $\mathbb{D}^{1,2,3,4}$ \\ ${ }^{1}$ Cell Therapy Center, Beijing Institute of Geriatrics, Xuanwu Hospital, Capital Medical University, Beijing 100053, China \\ ${ }^{2}$ Key Laboratory of Neurodegenerative Diseases, Ministry of Education, Beijing 100053, China \\ ${ }^{3}$ Center of Neural Injury and Repair, Beijing Institute for Brain Disorders, Beijing 100069, China \\ ${ }^{4}$ Center of Parkinson's Disease, Beijing Institute for Brain Disorders, Beijing 100069, China
}

Correspondence should be addressed to Zhiguo Chen; chenzhiguo@gmail.com

Received 15 February 2019; Accepted 7 April 2019; Published 5 May 2019

Guest Editor: Ming Li

Copyright (C) 2019 Sumei Liu and Zhiguo Chen. This is an open access article distributed under the Creative Commons Attribution License, which permits unrestricted use, distribution, and reproduction in any medium, provided the original work is properly cited.

\begin{abstract}
Endogenous neural stem cells (NSCs) exist in the central canal of mammalian spinal cords. Under normal conditions, these NSCs remain quiescent and express FoxJ1. After spinal cord injury (SCI), the endogenous NSCs of a heterogeneous nature are activated and proliferate and migrate towards the lesion site and mainly differentiate into astrocytes to repair the injured tissue. In vitro, spinal cord NSCs are multipotent and can differentiate into neurons, astrocytes, and oligodendrocytes. The altered microenvironments after SCI play key roles on the fate determination of activated NSCs, especially on the neuronal specification potential. Studies show that the activated spinal cord NSCs can generate interneurons when transplanted into the adult hippocampus. In addition, the spinal cord NSCs exhibit low immunogenicity in a transplantation context, thus implicating a promising therapeutic potential on SCI recovery. Here, we summarize the characteristics of spinal cord NSCs, especially their properties after injury. With a better understanding of endogenous NSCs under normal and SCI conditions, we may be able to employ endogenous NSCs for SCI repair in the future.
\end{abstract}

\section{Introduction}

Neural stem cells (NSCs) exist mainly in two regions in the adult central nervous system (CNS): brain and spinal cord [1-6]. NSCs remain quiescent under normal physiological conditions and can be activated under certain conditions such as CNS injury [7]. The activated NSCs can self-renew to maintain stem cell pool size and differentiate into neural cells for tissue repair. In this review, we will focus on the properties and behavior of endogenous spinal cord NSCs in normal situations and SCI.

\section{Endogenous Spinal Cord NSCs}

The identity of endogenous spinal cord NSCs has been debated in the past years. Astrocytes, oligodendrocyte progenitors, and ependymal cells have all been suggested as spinal cord stem cells previously by different groups [8-10].
Cortical astrocytes gain some NSC properties and assume a proliferative status after brain injury, but these cells cannot give rise to cell types other than astrocytes [11]. Some studies have suggested that oligodendrocyte progenitors can differentiate into astrocytic lineage in addition to oligodendrocytes, but recent studies failed to prove this and these cells seem to have a restricted potential for oligodendrocyte lineage only [12-14]. The only cell type that has been confirmed to be multipotent and retains a neurosphere-forming and passaging ability in vitro is ependymal cells [8]. What is noteworthy is that some parenchymal progenitors in regions other than the ependymal zone can proliferate after SCI and give rise to neurons and glia in vitro, but these cells can hardly be expanded in vitro (fewer than 2-3 passages) $[15,16]$.

2.1. Spinal Cord NSCs Lining the Central Canal. Ependymal cells lining the central canal are referred as spinal cord NSC niche. In the mouse spinal cord, ependymal cells originate 
at mid embryonic stages (E15.5) and completely surround the central canal by birth (P0) [17]. Based on studies on FoxJ1-CreER transgenic mice, the ependymal cells can be categorized into 3 basic types according to cell morphology rather than function: cuboidal ependymal cells, tanycytes, and radial ependymal cells [10]. Cuboidal ependymal cells are the most abundant multiciliated cells, and the radial ependymal cells are the less numerous type [18]. In adult macaque, there are 3 subtypes according to the number of cilia: uniciliated, biciliated, and multiciliated, and the first two subtypes give rise to new ependymal cells [19]. In addition, another cell type, cerebrospinal fluid- (CSF-) contacting neurons (CSF-CNs), exists in lower vertebrates, such as fish and amphibians but not in higher mammals such as dogs and cats $[20,21]$. NSCs extend an apical protrusion in the ependymal zone to contact with CSF; the flow of CSF can be sensed through a transmembrane sodium channel which can regulate the proliferation of these cells by activating Erk cascade [22].

2.2. Ependymal Cells Are Heterogeneous. Studies show that ependymal cells are heterogeneous and express radial glia cell markers such as RC1 and BLBP and NSC markers such as CD15 (also known as Lewis X antigen or SSEA-1), GFAP, PSA-NCAM, Musashi1, CD133/prominin-1, Sox2, Sox3, and Sox9, as well as vimentin and nestin [10, 23-26]. Nestin is expressed in dorsal and ventral poles of ependymal cells and CD15 and BLBP in dorsal regions [24]. In adult mouse spinal cord, the numbers of nestin-containing cells (NCCs) are the greatest in cervical vertebrae $1-7$, the second in thoracic $1-12$, and the smallest in lumbar $1-5$, and no significant difference exists in the left vs. right side [27].

\section{Response of Endogenous Spinal Cord NSCs after Injury}

Following injury, endogenous spinal cord NSCs go through 3 steps: activation, migration, and differentiation.

3.1. Spinal Cord NSC Activation. Studies have shown that spinal cord injury induces activation of spinal cord NSCs which otherwise would remain in a quiescent state or assume very slow proliferation under normal conditions [7, 28-30]. Ependymal cells, astrocytes, and NG2 ${ }^{+}$oligodendrocytes are all stimulated to divide after injury $[9,31,32]$. Spheres derived from injured spinal cords grow 3-4 times faster and larger than those from intact spinal cords [8]. Some ependymal cell markers such as Sox2, Sox3, and FoxJ1 are downregulated when the progeny cells leave the central canal and contribute to glial scar formation [10]. Another cell population called radial glia that express vimentin, BLBP, and nestin increase the expression of these markers after injury $[33,34]$. Reports show that SCI increases the number of Nestin ${ }^{+} / \mathrm{Sox} 2^{+}$cells in the spinal cord, especially in the central canal in rats [35-37]. The expression of nestin, which is a marker of NSCs, is upregulated in the central canal after acupuncture injury with a 25 G needle (Figure 1).

Changes of microenvironment, such as an increased level of certain soluble factors, a hypoxic condition due to vascular destruction, and immune responses, may contribute to NSC activation after CNS injury [38-43]. In human spinal cords, multipotent NSCs have been isolated and studied in vitro, and the number of $\mathrm{Nestin}^{+} / \mathrm{GFAP}^{+}$cells is increased after traumatic injury as examined by histopathological analysis of the spinal cord tissues [44-46]. Mitogenic agents such as EGF and FGF promote spinal cord NSC proliferation in vitro and in vivo, and EGF promotes migration from the central canal [47-49]. VEGF, whose expression level is increased after SCI, activates spinal cord NSC proliferation through VEGFR2 and EGFR signal pathways [37]. Spinal cord extracts of rats after SCI promote embryonic rat NSC proliferation in vitro through elevating Notch1 and Hes1 expression, and Notch pathway activation might be one component in the injury niche of the spinal cord that promotes NSC self-renewal [50].

3.2. Spinal Cord NSC Migration and Differentiation. Migration of the activated NSCs away from the central canal towards the lesion site can be detected by 3 days after spinal cord injury $[10,51]$. The migrating cells change their morphologies and lose expression of FoxJ1, Sox2, and Sox3 [10]. Reports show that the recruited NSCs at lesion sites mainly differentiate into astrocytes and, to a less degree, oligodendrocytes, but into no neurons after injury [10]. Yet controversy still exists in the field. Using Nestin-CreERT transgenic mice as a tracing model, Ren et al. and Zukor et al. indicated that ependymal cell-derived progenies contribute minimally to the protective scar-forming astrocytes [52, 53].

\section{Cellular Strategies for SCI Repair}

Spinal cord injury is a neurodegenerative disease that results in loss of neurons, astrocytes, and oligodendrocytes, leading to physical impairments $[54,55]$. In addition, inflammatory reactions, ischemia, and apoptosis cause secondary damage to spinal tissues. Cell therapy, or combined with the administration of growth factors and/or biomaterials, has shown promising potentials for SCI repair [56-61] (Figure 2). Transplantation of derivatives of embryonic cells, induced pluripotent stem cell (iPSC), NSC, or induced NSC (iNSC) has produced regenerative effect and partial recovery from injury [62-64].

Culture of mouse embryonic stem (ES) cells was first reported by Evans and Kaufman in 1981 [65] and human ES cells by Thomson and colleagues in 1998 [66]. ES cells can be easily propagated in vitro and form teratoma when injected in vivo; therefore, ES cells need to be differentiated into neural lineage precursors prior to transplantation. Studies have shown that neurally differentiated cells derived from mouse ES cells, when transplanted into a rat spinal cord 9 days after traumatic injury, could survive and differentiate into astrocytes, oligodendrocytes, and neurons and migrate as far as $8 \mathrm{~mm}$ away from the lesion boundary. Engraftment also improves the hind limb functions of the injured rats [67]. Transplantation of human ES-derived oligodendrocyte progenitor cells (OPCs) promotes remyelination and restores locomotor performance after SCI [68]. 


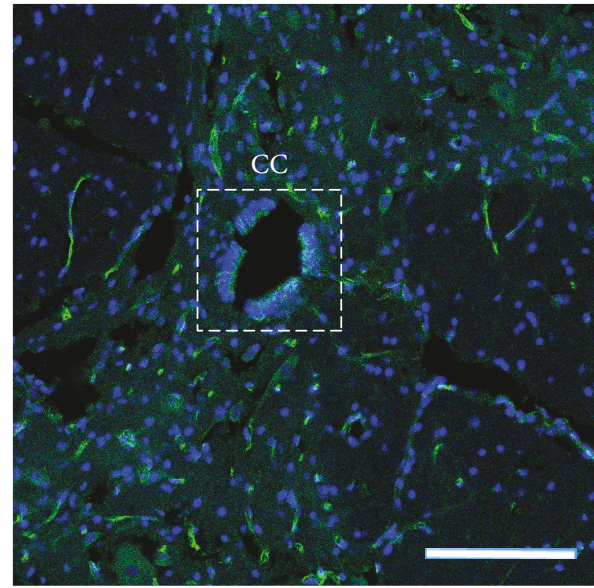

(a)

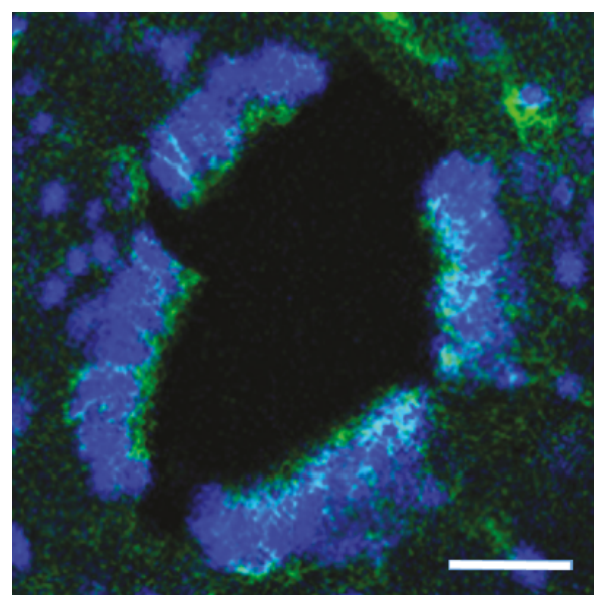

(c)

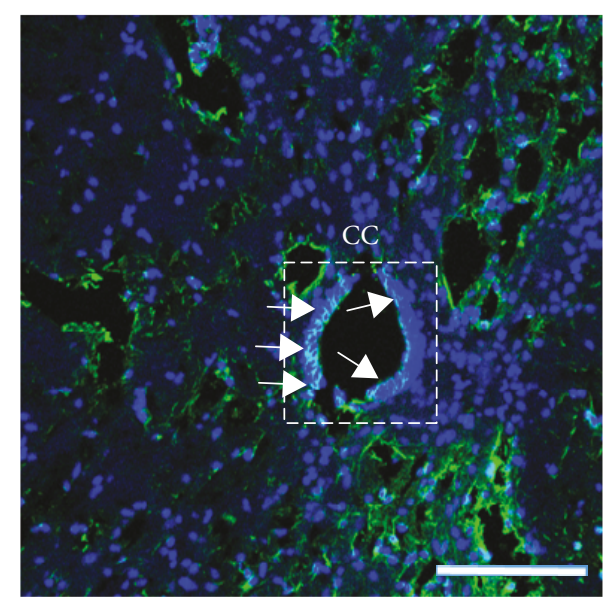

(b)

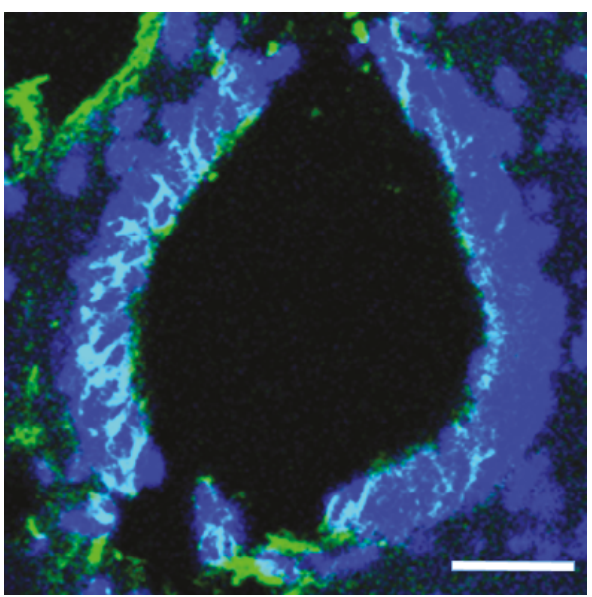

(d)

Figure 1: Transverse sections show an elevated expression of nestin in the rat central canal (CC). (a) Intact spinal cord. (b) 3 days after injury. ( $c$ and d) Magnified CC of the insets in (a) and (b), respectively. Nestin, green; DAPI, blue. Arrows show the Nestin ${ }^{+}$cells. Scale bars: (a and b): $200 \mu \mathrm{m}$; (c and d): $50 \mu \mathrm{m}$.

iPSCs are generated by a reprogramming process through overexpression of transcription factors such as Sox2, Klf4, Oct4, and c-Myc (SKOM) as reported by Takahashi and Yamanaka in 2006 [69]. Generation of iPSCs can also be realized via viral transduction, mRNA transfection, and/or small molecules [69-75]. iPSCs can be easily expanded to a large scale in culture and also of an autologous origin, thus circumventing most of the immune recognition-associated problems. However, a manufacturing scale-up process may affect the differentiation ability of iPSCs and thus the purity of desired derivatives accordingly; enrichment by applying immune-magnetic beads may be used to address this issue in some contexts. Researchers have grafted human iPSCderived neurospheres into the injured mouse spinal cords, and the grafts can form synapses and improve locomotor recovery [76, 77].

ES and iPSCs are pluripotent and can be differentiated into many lineages of cells, and thus have remarkable potentials to be used in a wide spectrum of conditions; however, the clinical application of ES cells is complicated by ethical problems; NSCs derived from embryos have similar issues in some countries, albeit to a less degree. Use of iPSCdifferentiated NSCs may circumvent these ethical issues. Researchers have examined embryonic NSCs in repair of SCI in animal models. Neurons derived from transplanted NSCs extracted from embryonic forebrains restore disrupted neuronal circuitry in mouse SCI models; nevertheless, another study shows that NSCs from the E14 rat cerebral cortex or the adult rat subventricular zone are restricted to a glial lineage when engrafted into the normal or lesioned spinal cord $[78,79]$. The seemingly difference between these two studies may be due to the fact that the former study transplanted embryonic NSCs together with valproic acid (VPA), which may promote neuronal differentiation from NSCs [79]. Okubo and colleagues have reported that iPSC-derived neural progenitor cells, together with gammasecretase inhibitors, promote functional recovery in the subacute and chronic phases of SCI $[80,81]$, and they are proposing an initiative to conduct a first-in-human clinical trial using hiPSC-NPCs to treat chronic SCI patients [82]. 


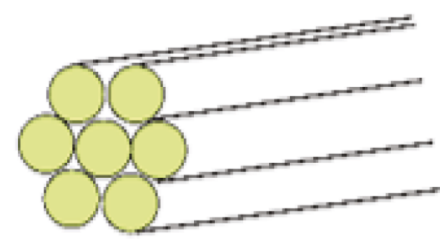

Aligned biomaterials
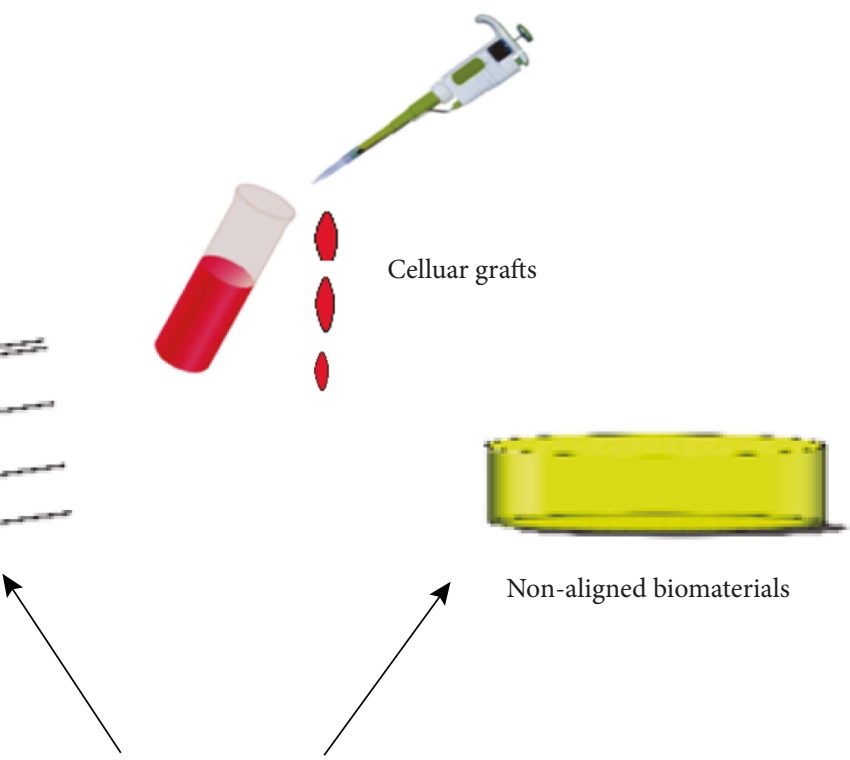

Non-aligned biomaterials

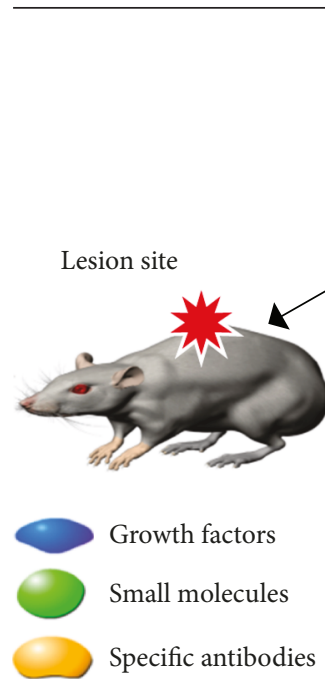

Cocktail
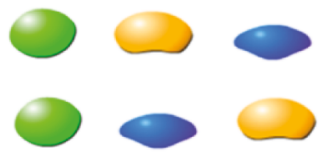

Specific antibodies

FIgUre 2: Combined cellular strategies for SCI repair.

\section{Potential of Endogenous Spinal Cord NSCs for SCI Repair}

Even though exogenous cell transplant may promote recovery after SCI, the carcinogenic risk, the invasive nature, and complications associated with transplantation procedures pose some challenges in the field. Furthermore, it is difficult to fully control the fate of the transplants [83]. Ependymal cells have been shown as the endogenous spinal cord NSCs. Some animals such as tailed amphibians exhibit powerful endogenous neurogenic capacity and are able to almost fully repair their damaged spinal cords and functionality after SCI [84-87]. Turtles spontaneously reconnect their severed spinal cords, leading in some cases to substantial recovery [88]. It has attracted a lot of interest with the idea that endogenous spinal cord NSCs might contribute to functional recovery. An in-depth understanding of the molecular mechanisms underlying the regeneration-permissive niches in these organisms may lend critical knowledge to help promote endogenous neurogenesis of the mammalian spinal cord after injury [89]. Also, it is reasonable to predict that, given the complicated nature of SCI, an individual approach targeting a single molecule/pathway may not be sufficient to offer a panacea, and tailoring specific combinations of therapies would provide a better outcome [90]. 
Ependymal cells differentiate into astrocytes to form scar tissues, and about half of the scar-associated astrocytes are derived from ependymal cells. The astrocytes migrate towards the core of the scar and produce laminin that is helpful for axon growth [91]. The GFAP ${ }^{+}$astrocytes derived from the resident astrocytes are localized in the brim of the scar and secrete chondroitin sulfate proteoglycans (CSPG) which are inhibitory to axon growth $[92,93]$. Eliminating ependymal cell-derived astrocytes or total reactive astrocytes in the scar tissue may enhance immune cell infiltration and lead to enlarged lesion volume, increased neuronal death, and aggravation of the functional outcome [94, 95]. Blocking the generation of ependymal cell progenies results in $79 \%$ animals failing to form compact scar tissues, secondary enlargement of lesions, and further axonal loss [96]. In addition, the recent studies report that astrocyte scar aids in axon growth, and RNA sequencing reveals that astrocytes and nonastrocytic cells in SCI lesions express multiple axon growth supporting molecules [97]. More interestingly, in fresh water turtles, the activated ependymal cells contributed to the generation of cycling cells that are an important part of the reconstructive bridging scaffold permissive for axon regrowth after SCI [98].

Isolation of ependymal cells from SCI rats and transplantation of these cells into severe contusion models lead to long-distance migration from the transplant bolus to the neurofilament-labeled axons in and around the lesion zone [99]. Retrovirus-mediated overexpression of the Neurogenin2 and Mash1, with growth factor treatment, enhances the production and maturation of new neurons and oligodendrocytes, when directly injected into the injured spinal cord [100]. Spinal cord NSCs also possess a property of plasticity. For example, adult spinal cord-derived stem cells that normally do not generate neurons after injury can differentiate into interneurons if injected into the adult hippocampus $[101,102]$. These studies indicate that ependymal cells have a potential to repair SCI, given sufficient conditions to manipulate the intrinsic properties of these cells and/or the surrounding microenvironment [103]. Furthermore, enhanced physical activity promotes the proliferation and differentiation of endogenous ependymal cells, indicating a key role of exercise on SCI recovery [104, 105]. Siegenthaler et al.'s work showed that voluntary exercise attenuates age-related reparative deficits following contusion SCI and the recovery rate of locomotor functions in injured aged rats is comparable to that of injured young rats without excise [106].

\section{Discussion}

There are 3 types of dividing cells in an intact spinal cord, $\mathrm{NG}^{+} / \mathrm{Olig}^{+}$oligodendrocyte progenitors, $\mathrm{GFAP}^{+} / \mathrm{CX} 30^{+} /-$ Sox $9^{+}$astrocytes, and Fox $1^{+}$ependymal cells, which constitute around $80 \%,<5 \%$, and $<5 \%$, respectively, of the dividing cells. Among these 3 types of cells, only ependymal cell-derived neurospheres are multipotent and can generate neurons, astrocytes, and oligodendrocytes in vitro. In vivo, ependymal cells are activated after injury and mostly differentiate into astrocytes and oligodendrocytes but few neurons.
The limited ability to differentiate into neurons may be partly due to the high expression of Notch1 and Hes1 in the niche after injury, which could be one of the components that inhibit neuronal differentiation [107]. A better understanding of the inhibitory microenvironment will help to find means to unleash the neuronal specification potential, both of the endogenous ependymal cells and of the incoming NSC transplants $[108,109]$. In addition, how the NSC transplants interact with the endogenous ependymal cells is not very clear and warrants a further study.

Previously, researchers have tried to transplant NSCs into the injured spinal cord immediately after SCI. However, this may not be the optimal time window for cellular intervention. Expression of inflammatory factors is increased in 6-12 hours after SCI and remains elevated in the following 4 days [110]. Astrocytes release various immune chemicals, such as CSPG, which is beneficial for acute SCI recovery but may be detrimental with chronic exposure, and MCP-1, which plays potent roles in the recruitment of macrophages and monocytes. The infiltrated neutrophils and macrophages can secrete myeloperoxidase, MMP-9, TNF- $\alpha$, TGF- $\beta$, IL- $\alpha$, IL- $1 \beta$, IL-6, IL-10, iNOS, Arg-1, etc. [111-113]. The inflammatory responses are the major cause of secondary tissue degeneration, namely, secondary SCI. In addition, neurotrophic factors, CNTF for example, which promotes differentiation into astrocytes, are increased after injury. However, factors that promote neuronal and oligodendrocytic genesis, such as NT3 and BDNF, remain at a low level. This contrasting expression of factors that block neurogenesis vs. enhance neurogenesis may be, at least in part, a reason for nonneuronal generation of endogenous NSCs after injury. It is possible that the optimal time for cellular intervention may not be the acute phase after injury. The secretion of inflammatory factors would last for 1 week, and the vascular reconstruction that is beneficial for neurogenesis occurs during 7-14 days [114]. Therefore, some researchers proposed that the optimal time window for cell transplantation might be 7-14 days postinjury [115].

The number of endogenous spinal cord NSCs is small in the central canal, despite their potential for SCI repair. A sufficient number of spinal cord NSCs may be essential to achieve certain reparative effects. To promote proliferation of endogenous NSCs, researchers have attempted to inject VEGF into spinal cords [37] or by electrical stimulation [116]. To promote the differentiation of endogenous NSCs into neurons, researchers have employed linearly organized biomaterials together with drugs such as cetuximab and taxol to repair the injured tissue [117]. However, more work is required to achieve a desirable level of activation and neuronal differentiation of endogenous spinal cord NSCs following SCI.

Furthermore, promoting both neurogenesis and oligogenesis is important for cell therapies for SCI repair. Some molecules or drugs, such as erythropoietin and cetuximab, have been reported to promote differentiation into neurons or oligodendrocytes [118-123]. Repression of the immune system in a balanced manner would also be conducive to recovery. For example, chondroitinase ABC (ChABC) delivery increases the digestion of CSPG and shifts the macrophage 
towards a M2 phenotype [124]; a single injection of rapamycin, a blocker of the mTOR pathway, reduces macrophage/ neutrophil infiltration and inhibits astrocyte activation, leading to increased neuronal survival and axonogenesis towards the lesion site [125]. Finding an optimal interventional strategy, possibly with a combinatory approach, to promote neurogenesis and oligogenesis and eventually reconstruct the damaged neuronal circuitry and functionality, is the goal in the field for SCI repair. Manipulating the intrinsic properties of ependymal cells at the central canal and turning the inhibitory niche at injury site to a permissive one, together with the exogenous application of NSC/neural precursor grafts and modulatory molecules/drugs, may be tailored to suit the complex conditions of individual patients with SCI in the future.

\section{Conflicts of Interest}

The authors declare that there is no conflict of interests.

\section{Acknowledgment}

This study received funding supports from the Stem Cell and Translation National Key Project (2016YFA0101403), the National Natural Science Foundation of China (81661130160, 81422014, 81561138004), the Beijing Municipal Natural Science Foundation (5142005), the Beijing Talents Foundation (2017000021223TD03), the Support Project of High-level Teachers in Beijing Municipal Universities in the Period of 13th Five-year Plan (CIT\&TCD20180333), the Beijing Medical System High Level Talent Award (2015-3-063), the Beijing Municipal Health Commission Fund (PXM 2018_026283_000002), the Beijing One Hundred, Thousand, and Ten Thousand Talents Fund (2018A03), the Beijing Municipal Administration of Hospitals Clinical Medicine Development of Special Funding Support (ZYLX201706), and the Royal SocietyNewton Advanced Fellowship (NA150482).

\section{References}

[1] B. Reynolds and S. Weiss, "Generation of neurons and astrocytes from isolated cells of the adult mammalian central nervous system," Science, vol. 255, no. 5052, pp. 1707-1710, 1992.

[2] S. Weiss, C. Dunne, and B. A. Reynolds, "Multipotent CNS stem cells are present in the adult mammalian spinal cord and ventricular neuroaxis," The Journal of Neuroscience, vol. 16, no. 23, pp. 7599-7609, 1996.

[3] F. Doetsch, I. Caillé, D. A. Lim, J. M. García-Verdugo, and A. Alvarez-Buylla, "Subventricular zone astrocytes are neural stem cells in the adult mammalian brain," Cell, vol. 97, no. 6, pp. 703-716, 1999.

[4] D. L. Stemple and D. J. Anderson, "Isolation of a stem cell for neurons and glia from the mammalian neural crest," Cell, vol. 71, no. 6, pp. 973-985, 1992.

[5] R. McKay, "Stem cells in the central nervous system," Science, vol. 276, no. 5309, pp. 66-71, 1997.

[6] D. Bottai, R. Fiocco, F. Gelain et al., "Neural stem cells in the adult nervous system," Journal of Hematotherapy \& Stem Cell Research, vol. 12, no. 6, pp. 655-670, 2003.
[7] C. B. Johansson, S. Momma, D. L. Clarke, M. Risling, U. Lendahl, and J. Frisén, "Identification of a neural stem cell in the adult mammalian central nervous system," Cell, vol. 96, no. 1, pp. 25-34, 1999.

[8] F. Barnabe-Heider, C. Goritz, H. Sabelström et al., "Origin of new glial cells in intact and injured adult spinal cord," Cell Stem Cell, vol. 7, no. 4, pp. 470-482, 2010.

[9] P. J. Horner, A. E. Power, G. Kempermann et al., "Proliferation and differentiation of progenitor cells throughout the intact adult rat spinal cord," The Journal of Neuroscience, vol. 20, no. 6, pp. 2218-2228, 2000.

[10] K. Meletis, F. Barnabe'-Heider, M. Carlén et al., "Spinal cord injury reveals multilineage differentiation of ependymal cells," PLoS Biology, vol. 6, no. 7, article e182, 2008.

[11] A. Buffo, I. Rite, P. Tripathi et al., "Origin and progeny of reactive gliosis: a source of multipotent cells in the injured brain," Proceedings of the National Academy of Sciences of the United States of America, vol. 105, no. 9, pp. 3581-3586, 2008.

[12] F. Guo, J. Ma, E. McCauley, P. Bannerman, and D. Pleasure, "Early postnatal proteolipid promoter-expressing progenitors produce multilineage cells in vivo," The Journal of Neuroscience, vol. 29, no. 22, pp. 7256-7270, 2009.

[13] S. H. Kang, M. Fukaya, J. K. Yang, J. D. Rothstein, and D. E. Bergles, "NG2+ CNS glial progenitors remain committed to the oligodendrocyte lineage in postnatal life and following neurodegeneration," Neuron, vol. 68, no. 4, pp. 668-681, 2010.

[14] S. Yoo and J. R. Wrathall, "Mixed primary culture and clonal analysis provide evidence that NG2 proteoglycan-expressing cells after spinal cord injury are glial progenitors," Developmental Neurobiology, vol. 67, no. 7, pp. 860-874, 2007.

[15] S. Yamamoto, N. Yamamoto, T. Kitamura, K. Nakamura, and M. Nakafuku, "Proliferation of parenchymal neural progenitors in response to injury in the adult rat spinal cord," Experimental Neurology, vol. 172, no. 1, pp. 115-127, 2001.

[16] F. Barnabe-Heider and J. Frisen, "Stem cells for spinal cord repair," Cell Stem Cell, vol. 3, no. 1, pp. 16-24, 2008.

[17] X. Li, E. M. Floriddia, K. Toskas, K. J. L. Fernandes, N. Guérout, and F. Barnabé-Heider, "Regenerative potential of ependymal cells for spinal cord injuries over time," EBioMedicine, vol. 13, pp. 55-65, 2016.

[18] J. Bruni and K. Reddy, "Ependyma of the central canal of the rat spinal cord: a light and transmission electron microscopic study," Journal of Anatonomy, vol. 152, pp. 55-70, 1987.

[19] C. Alfaro-Cervello, A. Cebrian-Silla, M. Soriano-Navarro et al., "The adult macaque spinal cord central canal zone contains proliferative cells and closely resembles the human," Journal of Comparative Neurology, vol. 522, no. 8, pp. 1800-1817, 2014.

[20] S. A. Moore, "The spinal ependymal layer in health and disease," Veterinary Pathology, vol. 53, no. 4, pp. 746-753, 2016.

[21] I. Vigh-Teichmann and B. Vigh, "The system of cerebrospinal fluid-contacting neurons," Archivum histologicum japonicum, vol. 46, no. 4, pp. 427-468, 1983.

[22] N. Kaneko and K. Sawamoto, "Go with the flow: cerebrospinal fluid flow regulates neural stem cell proliferation," Cell Stem Cell, vol. 22, no. 6, pp. 783-784, 2018.

[23] L. K. Hamilton, M. K. V. Truong, M. R. Bednarczyk, A. Aumont, and K. J. L. Fernandes, "Cellular organization of the central canal ependymal zone, a niche of latent neural 
stem cells in the adult mammalian spinal cord," Neuroscience, vol. 164, no. 3, pp. 1044-1056, 2009.

[24] H. Sabelstrom, M. Stenudd, and J. Frisen, "Neural stem cells in the adult spinal cord," Experimental Neurology, vol. 260, pp. 44-49, 2014.

[25] J.-C. Sabourin, K. B. Ackema, D. Ohayon et al., "A mesenchymal-like ZEB1+ niche harbors dorsal radial glial fibrillary acidic protein-positive stem cells in the spinal cord," Stem Cells, vol. 27, no. 11, pp. 2722-2733, 2009.

[26] U. Lendahl, L. B. Zimmerman, and R. D. G. McKay, "CNS stem cells express a new class of intermediate filament protein," Cell, vol. 60, no. 4, pp. 585-595, 1990.

[27] R. Xu, C. Wu, Y. Tao et al., "Nestin-positive cells in the spinal cord: a potential source of neural stem cells," International Journal of Developmental Neuroscience, vol. 26, no. 7, pp. 813-820, 2008.

[28] Y. Xu, M. Kitada, M. Yamaguchi, M. Dezawa, and C. Ide, "Increase in bFGF-responsive neural progenitor population following contusion injury of the adult rodent spinal cord," Neuroscience Letters, vol. 397, no. 3, pp. 174-179, 2006.

[29] Y. Ke, L. Chi, R. Xu, C. Luo, D. Gozal, and R. Liu, "Early response of endogenous adult neural progenitor cells to acute spinal cord injury in mice," Stem Cells, vol. 24, no. 4, pp. 1011-1019, 2006.

[30] L. L. Horky, F. Galimi, F. H. Gage, and P. J. Horner, "Fate of endogenous stem/progenitor cells following spinal cord injury," The Journal of Comparative Neurology, vol. 498, no. 4, pp. 525-538, 2006.

[31] D. M. McTigue, P. Wei, and B. T. Stokes, "Proliferation of NG2-positive cells and altered oligodendrocyte numbers in the contused rat spinal cord," The Journal of Neuroscience, vol. 21, no. 10, pp. 3392-3400, 2001.

[32] D. M. McTigue and F. R. Sahinkaya, "The fate of proliferating cells in the injured adult spinal cord," Stem Cell Research \& Therapy, vol. 2, no. 1, p. 7, 2011.

[33] A. Petit, A. D. Sanders, T. E. Kennedy et al., "Adult spinal cord radial glia display a unique progenitor phenotype," PLoS One, vol. 6, no. 9, article e24538, 2011.

[34] H. Nomura, H. Kim, A. Mothe et al., "Endogenous radial glial cells support regenerating axons after spinal cord transection," Neuroreport, vol. 21, no. 13, pp. 871-876, 2010.

[35] X. Li, Y. Zhao, S. Cheng et al., "Cetuximab modified collagen scaffold directs neurogenesis of injury-activated endogenous neural stem cells for acute spinal cord injury repair," Biomaterials, vol. 137, pp. 73-86, 2017.

[36] C. Fan, X. Li, Z. Xiao et al., "A modified collagen scaffold facilitates endogenous neurogenesis for acute spinal cord injury repair," Acta Biomater, vol. 51, pp. 304-316, 2017.

[37] S. M. Liu, Z. F. Xiao, X. Li et al., "Vascular endothelial growth factor activates neural stem cells through epidermal growth factor receptor signal after spinal cord injury," CNS Neuroscience \& Therapeutics, vol. 25, no. 3, pp. 375-385, 2018.

[38] Y. Yagita, K. Kitagawa, T. Sasaki et al., "Differential expression of Musashil and nestin in the adult rat hippocampus after ischemia," Journal of Neuroscience Research, vol. 69, no. 6, pp. 750-756, 2002.

[39] I. Mocchetti, S. J. Rabin, A. M. Colangelo, S. R. Whittemore, and J. R. Wrathall, "Increased basic fibroblast growth factor expression following contusive spinal cord injury," Experimental Neurology, vol. 141, no. 1, pp. 154-164, 1996.
[40] R. Covacu and L. Brundin, "Effects of neuroinflammation on neural stem cells," The Neuroscientist, vol. 23, no. 1, pp. 27-39, 2017.

[41] S. A. Frautschy, P. A. Walicke, and A. Baird, "Localization of basic fibroblast growth factor and its mRNA after CNS injury," Brain Research, vol. 553, no. 2, pp. 291-299, 1991.

[42] P. Follesa, J. R. Wrathall, and I. Mocchetti, "Increased basic fibroblast growth factor mRNA following contusive spinal cord injury," Molecular Brain Research, vol. 22, no. 1-4, pp. 1-8, 1994.

[43] C. Perez Estrada, R. Covacu, S. R. Sankavaram, M. Svensson, and L. Brundin, "Oxidative stress increases neurogenesis and oligodendrogenesis in adult neural progenitor cells," Stem Cells and Development, vol. 23, no. 19, pp. 2311-2327, 2014.

[44] A. Mothe and C. H. Tator, "Isolation of neural stem/progenitor cells from the periventricular region of the adult rat and human spinal cord," Journal of Visualized Experiments, no. 99, article e52732, 2015.

[45] C. Dromard, H. Guillon, V. Rigau et al., "Adult human spinal cord harbors neural precursor cells that generate neurons and glial cells in vitro," Journal of Neuroscience Research, vol. 86, no. 9, pp. 1916-1926, 2008.

[46] T. Cawsey, J. Duflou, C. S. Weickert, and C. A. Gorrie, "Nestin-positive ependymal cells are increased in the human spinal cord after traumatic central nervous system injury," Journal of Neurotrauma, vol. 32, no. 18, pp. 1393-1402, 2015.

[47] A. Kojima, "Epidermal growth factor and fibroblast growth factor 2 cause proliferation of ependymal precursor cells in the adult rat spinal cord in vivo," Journal of Neuropathology \& Experimental Neurology, vol. 59, no. 8, pp. 687-697, 2000.

[48] D. J. Martens, R. M. Seaberg, and D. Van Der Kooy, "In vivo infusions of exogenous growth factors into the fourth ventricle of the adult mouse brain increase the proliferation of neural progenitors around the fourth ventricle and the central canal of the spinal cord," European Journal of Neuroscience, vol. 16, no. 6, pp. 1045-1057, 2002.

[49] F. Gage, "Mammalian neural stem cells," Science, vol. 287, no. 5457, pp. 1433-1438, 2000.

[50] Q.-Z. Zhou, G. Zhang, H.-B. Long et al., "Effect of spinal cord extracts after spinal cord injury on proliferation of rat embryonic neural stem cells and Notch signal pathway in vitro," Asian Pacific Journal of Tropical Medicine, vol. 7, no. 7, pp. 562-567, 2014.

[51] A. J. Mothe and C. H. Tator, "Proliferation, migration, and differentiation of endogenous ependymal region stem/progenitor cells following minimal spinal cord injury in the adult rat," Neuroscience, vol. 131, no. 1, pp. 177-187, 2005.

[52] Y. Ren, Y. Ao, T. M. O'Shea et al., "Ependymal cell contribution to scar formation after spinal cord injury is minimal, local and dependent on direct ependymal injury," Scientific Reports, vol. 7, no. 1, article 41122, 2017.

[53] K. Zukor, S. Belin, C. Wang, N. Keelan, X. Wang, and Z. He, "Short hairpin RNA against PTEN enhances regenerative growth of corticospinal tract axons after spinal cord injury," Journal of Neuroscience, vol.33, no. 39, pp. 15350-15361, 2013.

[54] V. Sahni and J. A. Kessler, "Stem cell therapies for spinal cord injury," Nature Reviews Neurology, vol. 6, no. 7, pp. 363-372, 2010.

[55] M. S. Beattie, A. A. Farooqui, and J. C. Bresnahan, "Review of current evidence for apoptosis after spinal cord injury," Journal of Neurotrauma, vol. 17, no. 10, pp. 915-925, 2000. 
[56] J. Fan, H. Zhang, J. He et al., "Neural regrowth induced by PLGA nerve conduits and neurotrophin-3 in rats with complete spinal cord transection," Journal of Biomedical Materials Research Part B: Applied Biomaterials, vol. 97B, no. 2, pp. 271-277, 2011.

[57] P. Lu, "Chapter 1 - Stem cell transplantation for spinal cord injury repair," Progress in Brain Research, vol. 231, pp. 1-32, 2017.

[58] Q. Han, W. Jin, Z. Xiao et al., "The promotion of neural regeneration in an extreme rat spinal cord injury model using a collagen scaffold containing a collagen binding neuroprotective protein and an EGFR neutralizing antibody," Biomaterials, vol. 31, no. 35, pp. 9212-9220, 2010.

[59] M. Gao, P. Lu, B. Bednark et al., "Templated agarose scaffolds for the support of motor axon regeneration into sites of complete spinal cord transection," Biomaterials, vol. 34, no. 5, pp. 1529-1536, 2013.

[60] A. Raspa, R. Pugliese, M. Maleki, and F. Gelain, "Recent therapeutic approaches for spinal cord injury," Biotechnology and Bioengineering, vol. 113, no. 2, pp. 253-259, 2016.

[61] T. Yara, Y. Kato, H. Kataoka et al., "Environmental factors involved in axonal regeneration following spinal cord transection in rats," Medical Molecular Morphology, vol. 42, no. 3, pp. 150-154, 2009.

[62] A. J. Mothe and C. H. Tator, "Advances in stem cell therapy for spinal cord injury," The Journal of Clinical Investigation, vol. 122, no. 11, pp. 3824-3834, 2012.

[63] Q. Cao, R. L. Benton, and S. R. Whittemore, "Stem cell repair of central nervous system injury," Journal of Neuroscience Research, vol. 68, no. 5, pp. 501-510, 2002.

[64] M. G. Fehlings and R. Vawda, "Cellular treatments for spinal cord injury: the time is right for clinical trials," Neurotherapeutics, vol. 8, no. 4, pp. 704-720, 2011.

[65] M. Evans and M. Kaufman, "Establishment in culture of pluripotential cells from mouse embryos," Nature, vol. 292, no. 5819, pp. 154-156, 1981.

[66] J. Thomson, J. Itskovitz-Eldor, S. Shapiro et al., "Embryonic stem cell lines derived from human blastocysts," Science, vol. 282, no. 5391, pp. 1145-1147, 1998.

[67] J. W. McDonald, X. Z. Liu, Y. Qu et al., “Transplanted embryonic stem cells survive, differentiate and promote recovery in injured rat spinal cord," Nature Medicine, vol. 5, no. 12, pp. 1410-1412, 1999.

[68] H. S. Keirstead, G. Nistor, G. Bernal et al., "Human embryonic stem cell-derived oligodendrocyte progenitor cell transplants remyelinate and restore locomotion after spinal cord injury," The Journal of Neuroscience, vol. 25, no. 19, pp. 4694-4705, 2005.

[69] K. Takahashi and S. Yamanaka, "Induction of pluripotent stem cells from mouse embryonic and adult fibroblast cultures by defined factors," Cell, vol. 126, no. 4, pp. 663-676, 2006.

[70] H. Y. Choi, T. J. Lee, G. M. Yang et al., "Efficient mRNA delivery with graphene oxide-polyethylenimine for generation of footprint-free human induced pluripotent stem cells," Journal of Controlled Release, vol. 235, pp. 222-235, 2016.

[71] K. Li, S. Zhu, H. A. Russ et al., "Small molecules facilitate the reprogramming of mouse fibroblasts into pancreatic lineages," Cell Stem Cell, vol. 14, no. 2, pp. 228-236, 2014.

[72] L. Rohani, C. Fabian, H. Holland et al., "Generation of human induced pluripotent stem cells using non-synthetic mRNA," Stem Cell Research, vol. 16, no. 3, pp. 662-672, 2016.
[73] Y. Shi, C. Desponts, J. T. Do, H. S. Hahm, H. R. Schöler, and S. Ding, "Induction of pluripotent stem cells from mouse embryonic fibroblasts by Oct4 and Klf4 with smallmolecule compounds," Cell Stem Cell, vol. 3, no. 5, pp. 568574, 2008.

[74] A. Sridhar, S. K. Ohlemacher, K. B. Langer, and J. S. Meyer, "Robust differentiation of mRNA-reprogrammed human induced pluripotent stem cells toward a retinal lineage," Stem Cells Translational Medicine, vol. 5, no. 4, pp. 417-426, 2016.

[75] I. Wilmut, A. E. Schnieke, J. McWhir, A. J. Kind, and K. H. S. Campbell, "Viable offspring derived from fetal and adult mammalian cells," Nature, vol. 385, no. 6619, pp. 810-813, 1997.

[76] S. Nori, Y. Okada, A. Yasuda et al., "Grafted human-induced pluripotent stem-cell-derived neurospheres promote motor functional recovery after spinal cord injury in mice," Proceedings of the National Academy of Sciences of the United States of America, vol. 108, no. 40, pp. 16825-16830, 2011.

[77] N. Nagoshi and H. Okano, "iPSC-derived neural precursor cells: potential for cell transplantation therapy in spinal cord injury," Cellular and Molecular Life Sciences, vol. 75, no. 6, pp. 989-1000, 2017.

[78] Q. L. Cao, Y. P. Zhang, R. M. Howard, W. M. Walters, P. Tsoulfas, and S. R. Whittemore, "Pluripotent stem cells engrafted into the normal or lesioned adult rat spinal cord are restricted to a glial lineage," Experimental Neurology, vol. 167 , no. 1 , pp. $48-58,2001$.

[79] M. Abematsu, K. Tsujimura, M. Yamano et al., "Neurons derived from transplanted neural stem cells restore disrupted neuronal circuitry in a mouse model of spinal cord injury," J Clin Invest, vol. 120, no. 9, pp. 3255-3266, 2010.

[80] T. Okubo, A. Iwanami, J. Kohyama et al., "Pretreatment with a $\gamma$-secretase inhibitor prevents tumor-like overgrowth in human iPSC-derived transplants for spinal cord injury," Stem Cell Reports, vol. 7, no. 4, pp. 649-663, 2016.

[81] T. Okubo, N. Nagoshi, J. Kohyama et al., "Treatment with a gamma-secretase inhibitor promotes functional recovery in human iPSC- derived transplants for chronic spinal cord injury," Stem Cell Reports, vol. 11, no. 6, pp. 1416-1432, 2018.

[82] O. Tsuji, K. Sugai, R. Yamaguchi et al., "Concise review: laying the groundwork for a first-in-human study of an induced pluripotent stem cell-based intervention for spinal cord injury," Stem Cells, vol. 37, no. 1, pp. 6-13, 2019.

[83] E. Panayiotou and S. Malas, "Adult spinal cord ependymal layer: a promising pool of quiescent stem cells to treat spinal cord injury," Frontiers in Physiology, vol. 4, p. 340, 2013.

[84] N. Marichal, C. Reali, M. I. Rehermann, O. Trujillo-Cenóz, and R. E. Russo, "Progenitors in the ependyma of the spinal cord: a potential resource for self-repair after injury," in The Plastic Brain, R. Bernhardi, J. Eugenín, and K. Muller, Eds., vol. 1015 of Advances in Experimental Medicine and Biology, pp. 241-264, Springer, Cham, 2017.

[85] J. F. Diaz Quiroz and K. Echeverri, "Spinal cord regeneration: where fish, frogs and salamanders lead the way, can we follow?," Biochemical Journal, vol. 451, no. 3, pp. 353-364, 2013.

[86] N. R. Iyer, T. S. Wilems, and S. E. Sakiyama-Elbert, "Stem cells for spinal cord injury: strategies to inform differentiation and transplantation," Biotechnol Bioeng, vol. 114, no. 2, pp. 245-259, 2017.

[87] H. Yang, P. Lu, H. M. McKay et al., "Endogenous neurogenesis replaces oligodendrocytes and astrocytes after primate 
spinal cord injury," The Journal of Neuroscience, vol. 26, no. 8, pp. 2157-2166, 2006.

[88] M. I. Rehermann, N. Marichal, R. E. Russo, and O. TrujilloCenóz, "Neural reconnection in the transected spinal cord of the freshwater turtle Trachemys dorbignyi," The Journal of Comparative Neurology, vol. 515, no. 2, pp. 197-214, 2009.

[89] D. Lee-Liu, G. Edwards-Faret, V. S. Tapia, and J. Larraín, "Spinal cord regeneration: lessons for mammals from nonmammalian vertebrates," Genesis, vol. 51, no. 8, pp. 529-544, 2013.

[90] S. Thuret, L. D. Moon, and F. H. Gage, "Therapeutic interventions after spinal cord injury," Nature Reviews Neuroscience, vol. 7, no. 8, pp. 628-643, 2006.

[91] J. Frisén, C. B. Johansson, C. Török, M. Risling, and U. Lendahl, "Rapid, widespread, and longlasting induction of nestin contributes to the generation of glial scar tissue after CNS injury," The Journal of Cell Biology, vol. 131, no. 2, pp. 453-464, 1995.

[92] J. Silver and J. H. Miller, "Regeneration beyond the glial scar," Nature Reviews Neuroscience, vol. 5, no. 2, pp. 146-156, 2004.

[93] C. A. Gregoire, B. L. Goldenstein, E. M. Floriddia, F. BarnabéHeider, and K. J. L. Fernandes, "Endogenous neural stem cell responses to stroke and spinal cord injury," Glia, vol. 63, no. 8, pp. 1469-1482, 2015.

[94] M. Stenudd, H. Sabelstrom, and J. Frisen, "Role of endogenous neural stem cells in spinal cord injury and repair," JAMA Neurology, vol. 72, no. 2, pp. 235-237, 2015.

[95] J. R. Faulkner, J. E. Herrmann, M. J. Woo, K. E. Tansey, N. B. Doan, and M. V. Sofroniew, "Reactive astrocytes protect tissue and preserve function after spinal cord injury," Journal of Neuroscience, vol. 24, no. 9, pp. 2143-2155, 2004.

[96] H. Sabelstrom, M. Stenudd, P. Reu et al., "Resident neural stem cells restrict tissue damage and neuronal loss after spinal cord injury in mice," Science, vol. 342, no. 6158, pp. 637-640, 2013.

[97] M. A. Anderson, J. E. Burda, Y. Ren et al., "Astrocyte scar formation aids central nervous system axon regeneration," Nature, vol. 532, no. 7598, pp. 195-200, 2016.

[98] M. I. Rehermann, F. F. Santinaque, B. López-Carro, R. E. Russo, and O. Trujillo-Cenóz, "Cell proliferation and cytoarchitectural remodeling during spinal cord reconnection in the fresh-water turtle Trachemys dorbignyi," Cell and Tissue Research, vol. 344, no. 3, pp. 415-433, 2011.

[99] V. Moreno-Manzano, F. J. Rodríguez-Jiménez, M. GarcíaRoselló et al., "Activated spinal cord ependymal stem cells rescue neurological function," Stem Cells, vol. 27, no. 3, pp. 733-743, 2009.

[100] Y. Ohori, S. Yamamoto, M. Nagao et al., "Growth factor treatment and genetic manipulation stimulate neurogenesis and oligodendrogenesis by endogenous neural progenitors in the injured adult spinal cord," The Journal of Neuroscience, vol. 26, no. 46, pp. 11948-11960, 2006.

[101] L. S. Shihabuddin, P. J. Horner, J. Ray, and F. H. Gage, "Adult spinal cord stem cells generate neurons after transplantation in the adult dentate gyrus," The Journal of Neuroscience, vol. 20, no. 23, pp. 8727-8735, 2000.

[102] S. Temple, "The development of neural stem cells," Nature, vol. 414, no. 6859, pp. 112-117, 2001.

[103] C. G. Becker, T. Becker, and J. P. Hugnot, "The spinal ependymal zone as a source of endogenous repair cells across vertebrates," Progress in Neurobiology, vol. 170, pp. 67-80, 2018.
[104] D. Cizkova, M. Nagyova, L. Slovinska et al., "Response of ependymal progenitors to spinal cord injury or enhanced physical activity in adult rat," Cellular and Molecular Neurobiology, vol. 29, no. 6-7, pp. 999-1013, 2009.

[105] A. Foret, R. Quertainmont, O. Botman et al., "Stem cells in the adult rat spinal cord: plasticity after injury and treadmill training exercise," Journal of Neurochemistry, vol. 112, no. 3, pp. 762-772, 2010.

[106] M. M. Siegenthaler, N. C. Berchtold, C. W. Cotman, and H. S. Keirstead, "Voluntary running attenuates age-related deficits following SCI," Experimental Neurology, vol. 210, no. 1, pp. 207-216, 2008.

[107] Y. Liu, B. Tan, L. Wang et al., "Endogenous neural stem cells in central canal of adult rats acquired limited ability to differentiate into neurons following mild spinal cord injury," International Journal of Clinical Experimental Pathology, vol. 8, no. 4, pp. 3835-3842, 2015.

[108] N. Picard-Riera, B. Nait-Oumesmar, and A. Baron-Van Evercooren, "Endogenous adult neural stem cells: limits and potential to repair the injured central nervous system," Journal of Neuroscience Research, vol. 76, no. 2, pp. 223231, 2004.

[109] H. Okano, "Adult neural stem cells and central nervous system repair," in Stem Cells in Reproduction and in the Brain, J. Morser, S. I. Nishikawa, and H. R. Schöler, Eds., vol. 60 of Ernst Schering Res Found Workshop, pp. 215-228, Springer, Berlin, Heidelberg, 2006.

[110] J. Widenfalk, K. Lundströmer, M. Jubran, S. Brené, and L. Olson, "Neurotrophic factors and receptors in the immature and adult spinal cord after mechanical injury or kainic acid," The Journal of Neuroscience, vol. 21, no. 10, pp. 3457-3475, 2001.

[111] A. Ahmed, A. A. Patil, and D. K. Agrawal, "Immunobiology of spinal cord injuries and potential therapeutic approaches," Molecular and Cellular Biochemistry, vol. 441, no. 1-2, pp. 181-189, 2018.

[112] A. L. Bowes and P. K. Yip, "Modulating inflammatory cell responses to spinal cord injury: all in good time," Journal of Neurotrauma, vol. 31, no. 21, pp. 1753-1766, 2014.

[113] N. Kasinathan, M. Vanathi, V. Subrahmanyam, and J. Rao, "A review on response of immune system in spinal cord injury and therapeutic agents useful in treatment," Current Pharmaceutical Biotechnology, vol. 16, no. 1, pp. 26-34, 2015.

[114] H. Okano, Y. Ogawa, M. Nakamura, S. Kaneko, A. Iwanami, and Y. Toyama, "Transplantation of neural stem cells into the spinal cord after injury," Seminars in Cell \& Developmental Biology, vol. 14, no. 3, pp. 191-198, 2003.

[115] J. Madri, "Modeling the neurovascular niche: implications for recovery from CNS injury," Journal of Physiology and Pharmacology, vol. 4, pp. 95-104, 2009.

[116] Y. Huang, Y. Li, J. Chen, H. Zhou, and S. Tan, "Electrical stimulation elicits neural stem cells activation: new perspectives in CNS repair," Frontiers in Human Neuroscience, vol. 9, p. 586, 2015.

[117] C. Fan, X. Li, Y. Zhao et al., "Cetuximab and Taxol co-modified collagen scaffolds show combination effects for the repair of acute spinal cord injury," Biomaterials Science, vol. 6, no. 7, pp. 1723-1734, 2018.

[118] I. Hassouna, C. Ott, L. Wüstefeld et al., "Revisiting adult neurogenesis and the role of erythropoietin for neuronal and 
oligodendroglial differentiation in the hippocampus," Molecular Psychiatry, vol. 21, no. 12, pp. 1752-1767, 2016.

[119] F. F. Gonzalez, A. Larpthaveesarp, P. McQuillen et al., "Erythropoietin increases neurogenesis and oligodendrogliosis of subventricular zone precursor cells after neonatal stroke," Stroke, vol. 44, no. 3, pp. 753-758, 2013.

[120] X. Li, Z. Xiao, J. Han et al., "Promotion of neuronal differentiation of neural progenitor cells by using EGFR antibody functionalized collagen scaffolds for spinal cord injury repair," Biomaterials, vol. 34, no. 21, pp. 5107-5116, 2013.

[121] H. Zhang, X. Fang, D. Huang et al., "Erythropoietin signaling increases neurogenesis and oligodendrogenesis of endogenous neural stem cells following spinal cord injury both in vivo and in vitro," Molecular Medicine Reports, vol. 17, no. 1, pp. 264-272, 2018.

[122] B. T. Tan, L. Wang, S. Li, Z.-Y. Long, Y.-M. Wu, and Y. Liu, "Retinoic acid induced the differentiation of neural stem cells from embryonic spinal cord into functional neurons in vitro," International Journal of Clinical Experimental Pathology, vol. 8, no. 7, pp. 8129-8135, 2015.

[123] L. Yang, G. Li, J. Ye et al., "Substance P enhances endogenous neurogenesis to improve functional recovery after spinal cord injury," The International Journal of Biochemistry \& Cell Biology, vol. 89, pp. 110-119, 2017.

[124] K. Bartus, N. D. James, A. Didangelos et al., "Large-scale chondroitin sulfate proteoglycan digestion with chondroitinase gene therapy leads to reduced pathology and modulates macrophage phenotype following spinal cord contusion injury," Journal of Neuroscience, vol. 34, no. 14, pp. 48224836, 2014.

[125] Y. Goldshmit, S. Kanner, M. Zacs et al., "Rapamycin increases neuronal survival, reduces inflammation and astrocyte proliferation after spinal cord injury," Molecular and Cellular Neuroscience, vol. 68, pp. 82-91, 2015. 


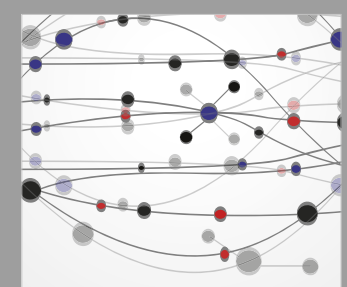

The Scientific World Journal
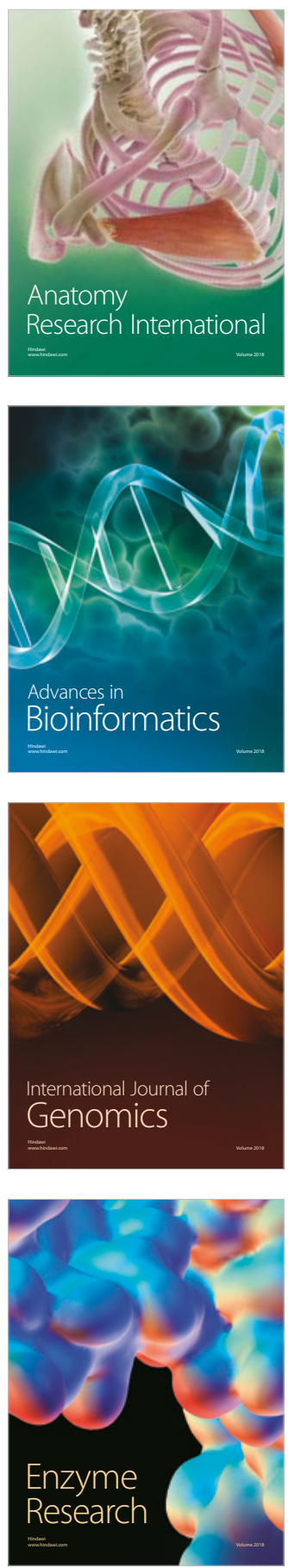
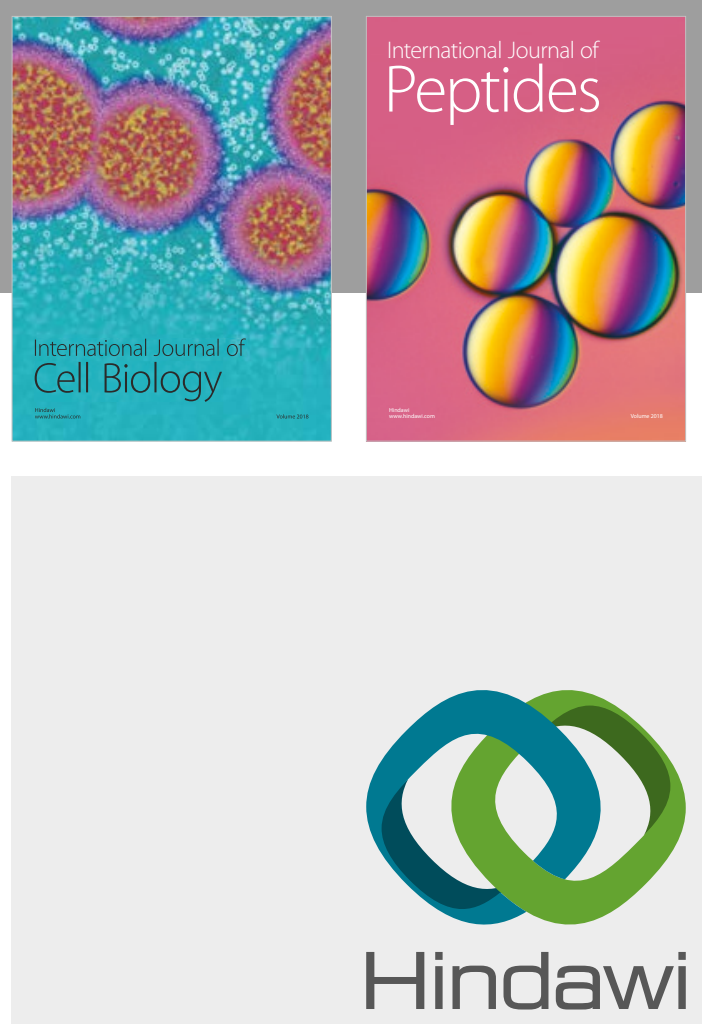

Submit your manuscripts at

www.hindawi.com
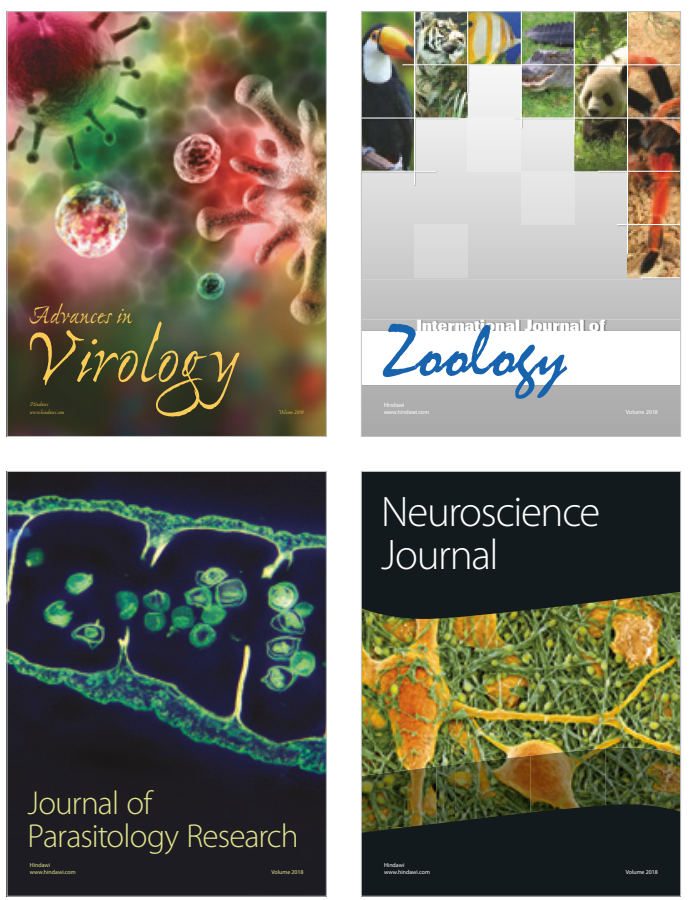
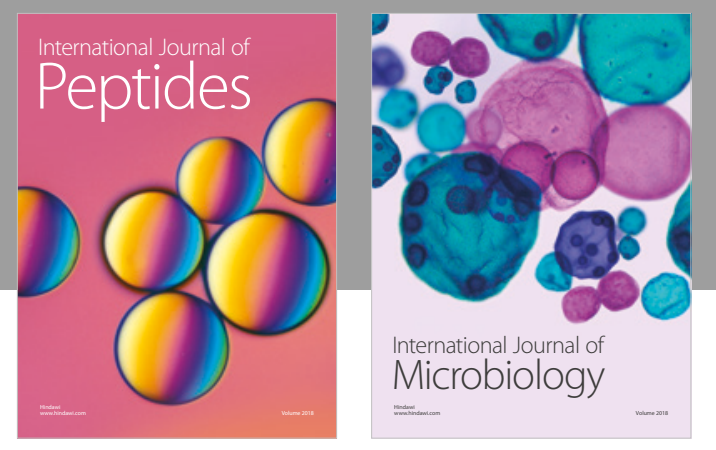

nternational Journal of Microbiology
Journal of
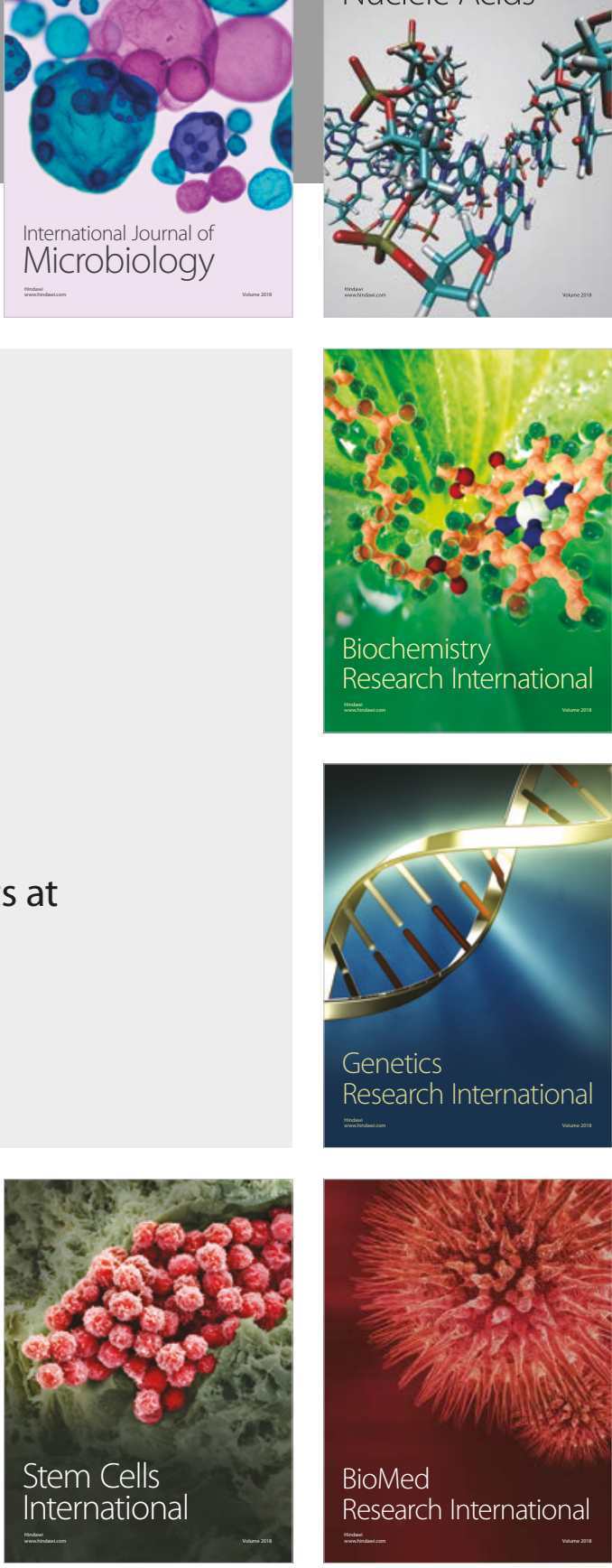
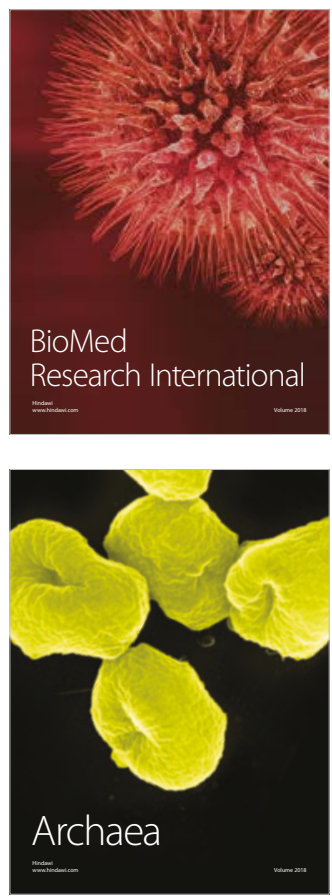